\title{
Polymer based microneedle patch fabricated using microinjection moulding
}

\author{
Pattanaphong Janphuang ${ }^{1, *}$, Mongkhol Laebua ${ }^{2}$, Chanwut Sriphung ${ }^{1}$, Phatsakon Taweewat ${ }^{2}$, Anan Sirichalarmkul ${ }^{2}$, \\ Kasiphisan Sukjantha ${ }^{2}$, Napatporn Promsawat ${ }^{1}$, Pimchanok Leuasoongnoen ${ }^{1}$, Somphong Suphachiaraphan ${ }^{1}$, \\ Kantapon Phimol ${ }^{1}$, Don Isarakorn ${ }^{3}$ and Somchai Tancharakorn ${ }^{1}$ \\ ${ }^{1}$ Synchrotron Light Research Institute (Public Organization), 111 Moo 6, University Avenue, Muang District, Nakhon Ratchasima, \\ 30000, Thailand \\ ${ }^{2}$ Thai-German Institute, 700/1 Moo 1 Amata Nakorn Industrial Estate, Bangna Trad Road, Klongtamru, Muang District, Chonburi, \\ 20000, Thailand \\ ${ }^{3}$ Department of Instrumentation and Control Engineering, Faculty of Engineering, King Mongkut's Institute of Technology Ladkrabang, \\ Bangkok, 10520, Thailand
}

\begin{abstract}
This paper presents the development of a polymer based microneedle patch for transdermal drug delivery application using plastic microinjection moulding. Design and analysis of the microneedle cavities and mould insert used in the injection moulding process were carried out using Computer-Aided Engineering (CAE) software. A mould insert with low surface roughness was fabricated using Micro Electrical Discharge Machining ( $\mu$-EDM). The injection moulding parameters including clamping force, temperature, injection pressure and velocity were characterized in order to obtain the optimum reproducibility. Solid truncated cone microneedles, made of biocompatible polymethyl methacrylate (PMMA), with a round tip radius of $50 \mu \mathrm{m}$ and $500 \mu \mathrm{m}$ in height have been realized by microinjection moulding process demonstrating the potential of a low cost, high production efficiency, and suitable for mass production. In addition, a mould insert of cylindrical microneedles fabricated using X-ray LIGA has been proposed.
\end{abstract}

\section{Introduction}

Currently, transdermal drug delivery (TDD) is one of a convenient way to deliver medicine into the subcutaneous tissues without drawbacks such as pain and digestive system disorders [1-2]. To achieve this, the drug is injected through the outermost layer of skin, Stratum Corneum (SC), from where it diffuses into the blood vessels of the deeper dermal skin layer through microneedle conduits. Since there are no nerves in SC layer, thus, this method is pain-free in comparison to hypodermic needle [3].

Microneedles (MNs) are micro-scale needles having thorn-like mechanical structure with at least one dimension of their shank smaller than $500 \mu \mathrm{m}$ [4]. The MNs are typically designed in arrays in order to improve the surface contact with the skin. Microneedle array technology is an emerging technique combining the ease of transdermal patch and the capability of hypodermic needle to facilitate penetration of actives into the skin. Various types of MNs yield have been developed since late of 1990s, including coated MNs enable drug on the surface of the needles, hollow MNs for drug infusion, and dissolving MNs encapsulated drug and release after insertion [5]. Previously, MNs were made of silicon, metals, and organic materials fabricated using laser ablation, wet etching, and metal electrodeposition processes [1]. In recent years, polymers based MNs is of great interest. Most of polymers have high toughness and biocompatible enabling polymers based MNs to avoid crack and hardly induce side effect during their insertion into the skin. Some polymers are water-soluble or biodegradable. Thus, drugs can be encapsulated in dissolvable MNs. Moreover, due to the relatively low melting temperature, microinjection moulding can be used in preparation of polymer MNs. Injection molding is a process in which melt materials, mostly polymer, is forced to flow under high pressure through a plunger into a mold cavity, where it solidifies and then ejected to produce a replicated structure on the surface. Thus, mould based fabrication technique could further reduce the manufacturing cost and is suitable for mass production.

Here, we present the design and fabrication method for an inexpensive microneedle patch made of biocompatible polymer, PMMA, by means of plastic microinjection moulding process. A detailed description on the flow analysis of molten polymer into the cavity, driven by the pressure applied by the plunger was studied through simulation in Moldex3D and $\mu$-EDM was used to fabricate the mould insert. By optimizing the injection moulding parameters, results show that low surface roughness polymer based microneedle patch can be produced. In addition, a mould insert of microneedle patch fabricated using X-ray LIGA are proposed.

${ }^{*}$ Corresponding author: pattanaphong@ $@$ slri.or.th 


\section{Design and fabrication}

\subsection{Microneedle design}

The feature of MNs should meet several requirements in order to achieve painless penetration into skin. The MNs should be sharp and strong enough to handling induced mechanical failure during the insertion of MNs into skin. The MNs should have proper length and diameter to avoid nerve contact and the short-shot during the microinjection moulding process. As illustrated in [6], considering the skin deformation, the MNs shank length is limited to 300 $-400 \mu \mathrm{m}$. However, it can be assumed that the entire needle shank will not penetrate into the skin since the human skin is very flexible. Thus, the length of the MNs in this work is defined to be $500 \mu \mathrm{m}$. Figure 1 shows the schematic diagram and dimensions of the designed microneedle. The material properties are given in table 1 .

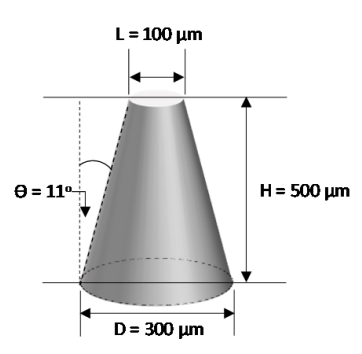

(a)

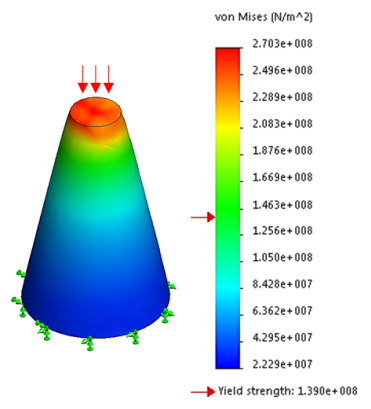

(b)
Fig. 1. (a) Geometric shape of a microneedle (MN); (b) Simulated stress and deformation of the MN under applied pressure of $300 \mathrm{MPa}$ on the tip.

The designed MN in this study is solid truncated cone structure, as illustrated in Fig. 1(a), in order to minimize the bending stress and detrimental buckling failure during the insertion of MN into the skin compared with other high aspect ratio or column-like structures [1]. It is obvious that sharpening tip, by reducing the tip diameter (L) and increasing the bevel angle $(\Theta)$, can effectively diminish the insertion force. However, it may result a tip damage during the penetration. Thus, a trade-off between sharpness and mechanical strength of the tip should be taken into account.

Table 1. PMMA material properties used in simulation [7].

\begin{tabular}{|l|c|}
\hline Properties & $8.30 \mathrm{GPa}$ \\
\hline Elastic modulus & 0.28 \\
\hline Poisson ratio & $3.20 \mathrm{GPa}$ \\
\hline Shear modulus & $1400 \mathrm{~kg} \mathrm{~m}^{-3}$ \\
\hline Density & $1.43 \mathrm{GPa}$ \\
\hline Tensile strength & $1.39 \mathrm{GPa}$ \\
\hline Yield strength & \\
\hline
\end{tabular}

The designed $\mathrm{MN}$ was then computer simulated in SolidWorks in order to determine the stress distribution and deformed shape of the MN. Fig. 1(b) illustrates the stress developed on the MN under applied pressure of 300 MPa to the tip of the MN. It is clearly seen that the tip could endure the applied pressure but the $\mathrm{MN}$ is deformed at this point. As described in [7], a pressure of $15 \mathrm{MPa}$ is sufficient for the penetration of human skin by liquid jet, diameter of 0.1-0.5 $\mathrm{mm}$. The penetration of microneedle requires less pressure than high-speed liquid jet. Consequently, we can estimate that the designed $\mathrm{MN}$ should not deformed in the human skin during the insertion. To evaluate the capability of microinjection moulding process in this work, $400 \mathrm{MNs}$ made of PMMA with $1 \mathrm{~mm}$ pitch between needles will be fabricated.

\subsection{Mould design}

The CAD model of a mould insert (or cavity insert) is illustrated in Fig. 2. It consists of 400 microneedle cavities (Fig. 2(b)) occupying approximately $500 \mathrm{~mm}^{2}$. The thickness of the base portion is designed to be $2 \mathrm{~mm}$. In order to successfully fabricate MNs by microinjection moulding process, guidelines for the design of a runner system and a detailed description on the flow analysis of molten polymer into the cavity were carried out by FE simulation in Moldex3D.

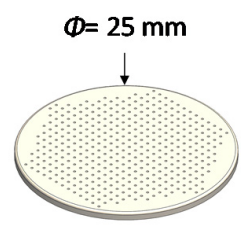

(a)

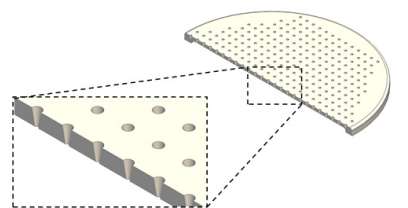

(b)
Fig. 2. (a) Schematic diagram of the mould insert designed for plastic injection of microneedle patch; (b) Cross-sectional view of the mould insert.

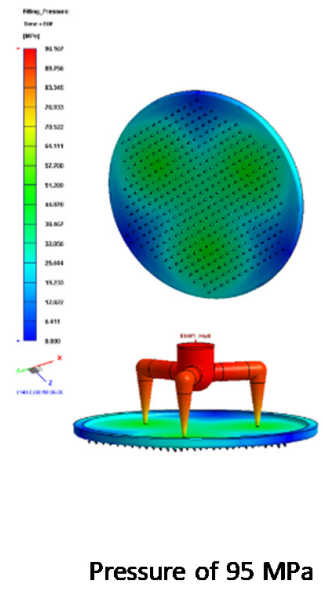

(a)

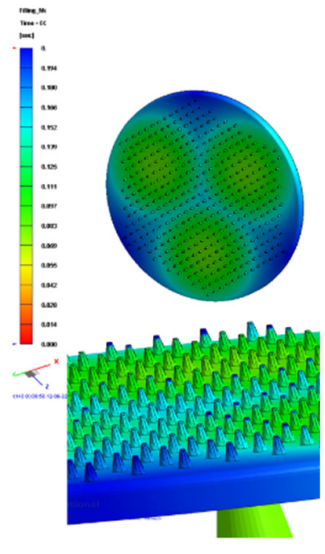

Filled in $0.2 \mathrm{sec}$.

(b)
Fig. 3. Simulated results of (a) injection pressure loaded to the designed runners and the mould insert and (b) molten PMMA filled in the cavity.

The runner optimization analysis is first performed in order to define the size and the number of runners, and the injection pressure applied to the cavity. To assure symmetric filling, pin point gate type was designed. In 
simulation, polymer melt temperature $\left(T_{p}=230{ }^{\circ} \mathrm{C}\right)$, mould temperature $\left(T_{m}=50{ }^{\circ} \mathrm{C}\right)$, holding pressure $\left(P_{h}=\right.$ $60 \mathrm{MPa})$, and injection flow $\left(V_{i}=9 \mathrm{ccm} / \mathrm{s}\right)$ were kept constant (to the values recommended by the manufacturer). The simulated results revealed that threepoint gate, with diameter of $5 \mathrm{~mm}$, were sufficient for the injection of molten PMMA, as shown in Fig. 3. More than $90 \%$ of MNs cavities were fulfilled by the molten PMMA in $0.2 \mathrm{~s}$ using injection pressure of $95 \mathrm{MPa}$ and gate freeze time of $0.38 \mathrm{~s}$, as illustrated in Fig. 3(b). Both fill and demoulding of high aspect ratio structure like microneedle are likely to be difficult. Air could be trapped inside the cavities blocking the filling process. The vacuum condition could be introduced to the system in order to improve the injection moulding capability.

\subsection{Fabrication of the mould insert}

The MN cavities in the mould insert were fabricated using $\mu$-EDM (SARIX SX-100, Switzerland). The whole MN mould was mainly made of high temperature alloy (SKD11) in order to enhance the corrosion resistance and wear resistance with less thermal expansion stress. The basic working principle of $\mu$-EDM process is described in Fig. 4.

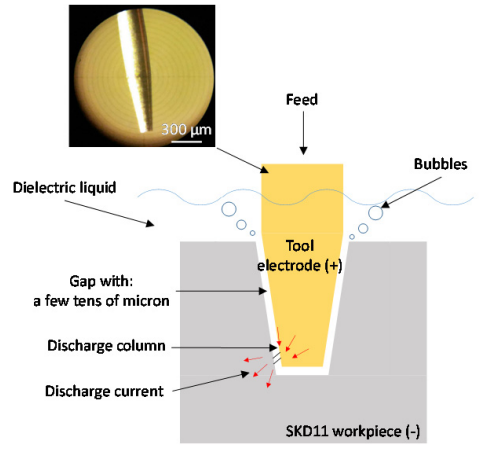

(a)

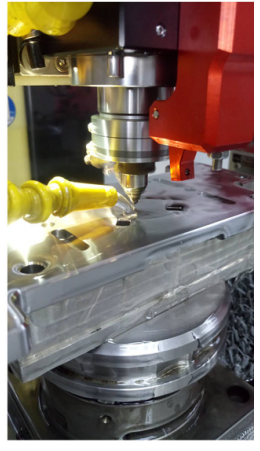

(b)
Fig. 4. (a) Schematic diagram of $\mu$-EDM process; (b) Machining of mould insert by $\mu$-EDM.

To produce the MN cavities, a submerging of the anode-cathode system into a dielectric liquid is required (Fig 4(a)). In $\mu$-EDM process, the designed MN structure was firstly created on a brass tool electrode using $100 \mu \mathrm{m}$ solid carbide end milling tools from SARIX. The material was then removed from the workpiece electrode by current discharges between two electrodes subjected to a voltage. When the voltage was increased, the electric field in the volume between two electrodes become larger than the strength of the dielectric, resulting in the breakdown of the dielectric allowing current to flow between electrodes. Consequently, the workpiece was then eroded and formed a replica of the tool electrode on the workpiece by a thermal process under a controlled electric spark [8]. During the fabrication, the open gap voltage between the electrodes was maintained at $100 \mathrm{~V}$ until the dielectric broke down. Figure 5 shows optical images of the fabricate mould insert fabricated by $\mu$-EDM. It should be noted that mould insert surfaces in which molten polymer undergoes have to be very smooth in order to avoid sticking of polymer during the demoulding process.

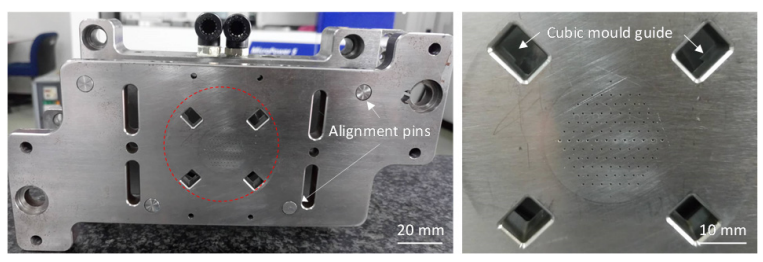

Fig. 5. Optical images of the MNs mould insert made of SKD11 alloy fabricated using precision $\mu$-EDM.

\section{Microinjection moulding}

The microinjection mould insert was carefully aligned inside the mould base through the alignment pins and the mould guide to ensure proper matching of the cavities. The mould insert and mould based were then installed into the MircroPower-5t microinjection moulding machine (Wittmann Battenfeld, Austria), as shown in Fig. 6, to investigate the capability of microinjection moulding process for the production of microstructure.

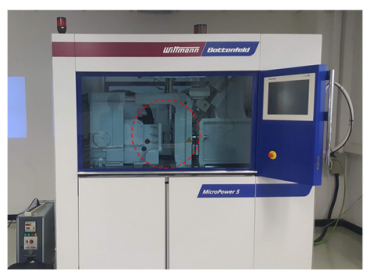

(a)

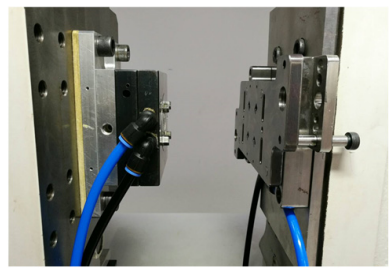

(b)
Fig. 6. Optical images of (a) MicroPower-5t microinjection moulding machine and (b) the installed microneedle mould.

The plunger diameter of the machine is set to be $5 \mathrm{~mm}$. During the microinjection moulding process, injection moulding parameters such as shot size, temperature (melt and mould), injection flow, clamping force, injection and holding pressure have been characterized in order to optimize the moulding results and reproducibility. Details of microinjection moulding parameters are described in table 2 .

Table 2. Microinjection moulding parameters.

\begin{tabular}{|l|c|}
\hline Parameters & \\
\hline Shot size & $1 \mathrm{ccm}$ \\
\hline Melt temperature $\left(T_{p}\right)$ & $230^{\circ} \mathrm{C}\left(\max .250^{\circ} \mathrm{C}\right)$ \\
\hline Mold temperature $\left(T_{m}\right)$ & $50^{\circ} \mathrm{C}\left(\max .70^{\circ} \mathrm{C}\right)$ \\
\hline Injection flow $\left(V_{i}\right)$ & $9 \mathrm{ccm} / \mathrm{s}$ \\
\hline Injection pressure $\left(P_{i}\right)$ & $120 \mathrm{MPa}$ \\
\hline Holding pressure $\left(P_{h}\right)$ & $60 \mathrm{MPa}$ \\
\hline Clamping force & $5000 \mathrm{~kg}$ \\
\hline Cooling duration & $9 \mathrm{~s}$ \\
\hline
\end{tabular}

The machine temperature was set at $230{ }^{\circ} \mathrm{C}$ to maintain the PMMA in the molten state. The shot size of $1 \mathrm{ccm}$ was selected. Using large shot size (greater than 1 $\mathrm{ccm}$ ), flush will occur. The clamping force was 5 tons $(5000 \mathrm{~kg})$ and the injection pressure was found to be 120 $\mathrm{MPa}$. Both of these parameters are close to the maximum 
capacity of the MicroPower-5t. Complete polymer based microneedle patch fabricated using microinjection moulding is illustrated in Fig. 7.

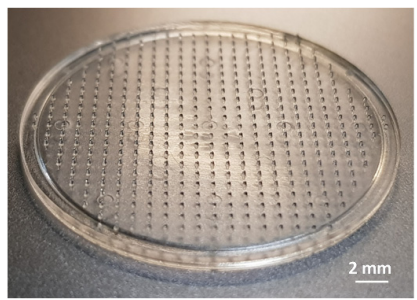

(a)

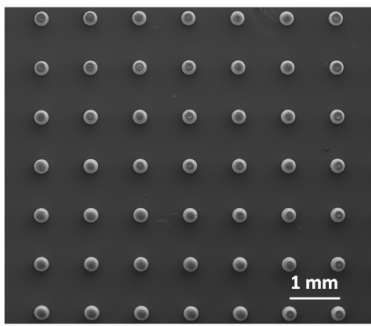

(c)

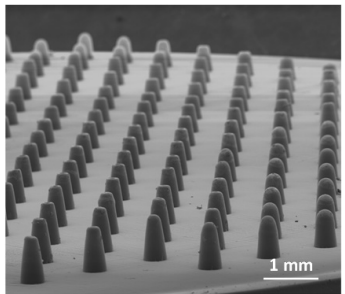

(b)

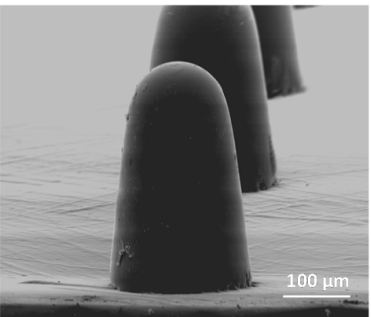

(d)

Fig. 7. (a) Optical images of a fabricated microneedle patch, and SEM images of (b) MNs array, (c) top view of the MNs array, and (d) a close-up of a MN.

\section{Fabrication of microneedle mould insert using $X$-Ray LIGA}

LIGA, a German acronym for Lithographie, Galvanoformung, and Abformung) process utilizes deep $\mathrm{x}$-ray exposure for the fabrication of high aspect ratio microstructure. Using high-energy photons, which is deep x-ray, microneedle shank can be fabricated. However, LIGA process is a planar exposure technique to construct 3D microstructure. Thus, a vertical sidewall microstructure could be achieved. In this section, we present a fabrication of MN mould insert using x-ray LIGA and the potential of using $\mathrm{x}$-ray exposure for the fabrication of sharp tipped microneedle is proposed.

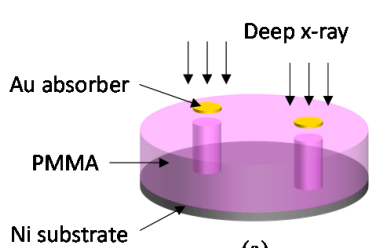

(a)

Electrodeposited $\mathrm{Ni}$

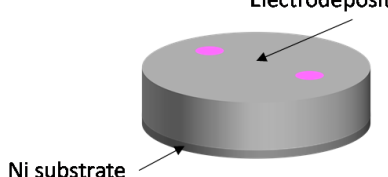

(c)

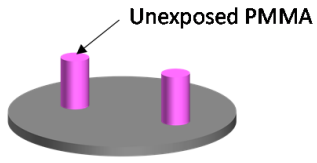

(b)

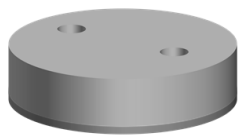

(d)
Fig. 8. Schematic diagram of the microfabrication process for the MNs mould insert using x-ray LIGA. (a) Vertical deep x-ray exposure; (b) Development of column-like microneedles; (c) Electrodeposition of Ni; and (d) PMMA stripping.
As shown in Fig. 8, the unexposed PMMA area blocked by gold (Au) absorber (Fig. 8(a)) making high aspect ratio column-like (cylindrical) MNs using deep x-ray produced by synchrotron radiation (Fig. 8(b)). $500 \mu \mathrm{m}$-thick Nickel (Ni) is then electrodeposited directly on top of $\mathrm{Ni}$ substrate (Fig. 8(c)). After stripping PMMA, the Ni mould insert is achieved (Fig. 8(d)). Figure 9 shows Ni the mould insert fabricated using $\mathrm{x}$-ray LIGA.

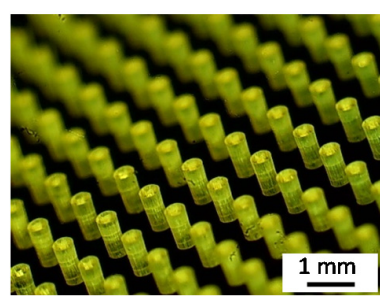

(a)

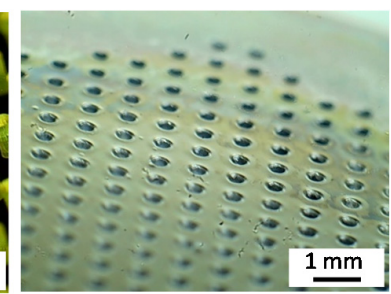

(b)
Fig. 9. Optical image of (a) cylindrical MNs based on PMMA exposed by deep $\mathrm{x}$-ray and (b) Ni mould insert fabricated using x-ray LIGA.

Using several combinations of deep x-ray exposure conditions including deep x-ray exposure angle (exposure of an inclined plane), and a gap between the gold absorber mask and PPMA, sharp tipped MNs made of PMMA can be realized.

\section{Conclusions}

Polymer based microneedle patch fabricated using microinjection moulding has been successfully demonstrated in this paper. Using this technique, the mass production of microneedle patch can be realized. The design of the microneedle architecture to avoid the bedof-nail effect, and penetration testing experiments are ongoing for the optimization of the transdermal drug delivery using microneedle.

\section{References}

1. G. Ma, Ch. Wu. J Control Release 251, 11-23 (2017)

2. M.R. Prausnitz, R. Langer. Nat. Biotechnol 26, 12611268 (2008)

3. S. Indermun, R. Luttge, Y.E. Choonara, P. Kumar, L.C. du Toit, G. Modi, V. Pillay. J. Control. Release 185, 130-138 (2014)

4. B. Stoeber, D. Liepmann. JMEMS 14, $472-479$ (2005)

5. E. Larraneta, R.E.M Lutton, A.D. Woolfson, R.F. Donnelly. Mater. Sci. Eng. R. Rep 104, 1-32 (2016)

6. S.J. Moon, S.S. Lee. J. Micromech. Microeng 15, 903 911 (2005)

7. K.L. Yung, Ch. Kang, H. Liu, K.F. Tam, S.M. Ko, F.Y Kwan, T.M.H. Lee. J. Micromech. Microeng 22, 015016 (2012)

8. K. Maghsoudi, R. Jafari, G. Momen, M. Farzaneh. Mater. Today. Commun. 13, 126-143 (2017) 